\title{
Development of Adaptive E-Learning Course in Moodle System
}

\author{
Vladislav Kukartsev ${ }^{1,2}$, Ekaterina Chzhan ${ }^{1}$, Vadim Tynchenko ${ }^{1,2^{*}}$, Oleslav Antamoshkin ${ }^{1,2,3}$, Alena Stupina ${ }^{1,2,3}$ \\ ${ }^{1}$ Siberian Federal University, 79, Svobodny pr., Krasnoyarsk, 660041, Russia \\ ${ }^{2}$ Siberian State Aerospace University, 31, Krasnoyarsky Rabochy Av., 660037 Krasnoyarsk, Russia \\ ${ }^{3}$ Krasnoyarsk State Agrarian University, 90, Mira pr., 660049, Krasnoyarsk, Russia
}

\begin{abstract}
E-learning has been actively developing recently. To date, there are a large number of platforms and developed courses. The main drawback is the lack of adaptability. This paper presents the results of developing an adaptive e-learning course on the Moodle platform. The course is based on a tree of concepts and discipline operations; the course content is based on different standards. Training takes place along various trajectories depending on the characteristics and level of the student's preparation, which will improve the learning achievement of students. The results of the development and implementation of an adaptive electronic course designed for students of the second year of full-time education are presented. In the process of learning, students follow a certain trajectory; on each trajectory, different material is represented.
\end{abstract}

\section{Introduction}

Today, electronic educational courses (EEC) are actively introduced. Demand and the need for electronic courses can be explained by the following factors [1-4].

First, educational technologies must be adequate to modern realities [3, 5-7]. Modern technologies have found wide application in various fields [6, 8, 9], undoubtedly the use of advances in informatization and computerization in teaching. Training in the usual mode using only boards and chalk looks somewhat an outdated model.

Secondly, the use of EEC makes learning more accessible. So, you can study the material not only in the walls of the audience, but in any place, having a computer or a mobile device and access to the Internet. Unconditional plus is the development of students' independence. Students plan the time and duration of classes.

One of the main didactic problems of the educational process is an individual approach to each student. This requirement is difficult for traditional (face-to-face) teaching methods. On the contrary, e-learning makes it possible to apply an individual approach to students, which is an undeniable advantage over traditional methods.

If in the early years of EEC development, the "individual approach" was understood as the different time that the student spent studying the material, today it is possible to implement and provide a choice of an individual trajectory of learning material. This kind of EEC is called adaptive - the course can be adjusted to the individual characteristics and preferences of the learner.
When using individual face-to-face trajectories, students have problems with assessing real achievements, productivity, and most importantly, by choosing the most suitable trajectory of learning $[10,11]$.

The peculiarity of adaptive EOK is that the regulation of the rate of mastering the material, as well as the initial choice of the trajectory, takes place without the participation of the instructor in the automatic mode $[4,5$, 12-14].

An important stage in the development of the course is the development of training trajectories. Trajectories are closely related to the metadata of the learning object (LO) [5].

The standard [9] defines five values for the difficulty of the LO: very light, light, medium, difficult, very difficult (very easy, easy, medium, difficult, very difficult). The authors of [5] speak of the sufficiency of three trajectories: high, medium and low, and their correspondence to the five-level type of metadata.

The relevance of using this approach in the implementation of e-courses can be judged by the results of a six-year project to monitor opportunities for realizing the potential of young people in the scientific and technical sphere conducted by the Center for Monitoring Technological Modernization and Scientific and Technological Development. As a result of the research, 14 reasons were identified that form a "new" education, in the first place - adaptive learning.

Adaptive learning is understood as the use of computers, mobile devices in the learning process, with the possibility of adapting the material to the needs of students. 
The article deals with the implementation and use of adaptive EEC, developed for full-time students.

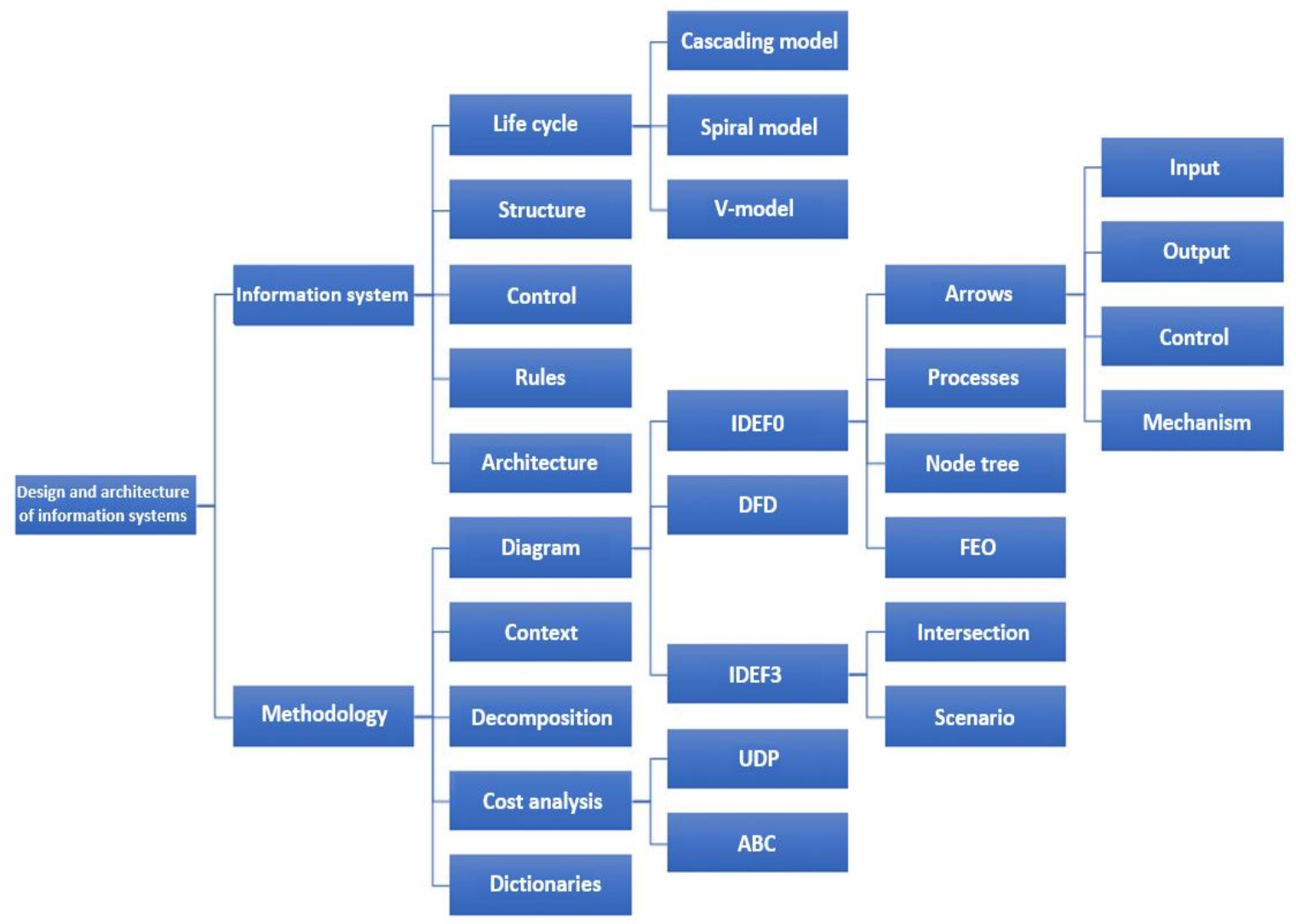

Fig. 1. Tree of concepts.

\section{Structure of electronic educational course}

When developing an electronic course (adaptive or traditional), special attention is paid to the theoretical material that underlies the course. The course content is based on the following normative documents: the federal state educational standard, professional and international standards. The current standard does not clearly regulate the content of the course (the topic of the lecture material); then the courses take into account both previous standards and future ones that have already been made public, but will come into force in the near future.

The material in the EEC consists of several logically interconnected sections or modules. One way of structuring a material is to compile a tree of concepts. As an example, in Fig. 1 the tree of concepts of the course "Design and architecture of information systems" is presented: tree tops are concepts; connecting lines are logical links between them.

In the framework of training with the electronic educational course, students learn not only theoretical material, but also acquire some skills, performing various tasks. The development of tasks for practical and seminar classes takes place according to the following tree of operations (Fig. 2).
As can be seen from the scheme, the course is based on two basic concepts - the information system and methodology.

The main problem of the presentation of material in a standard electronic course is not taking into account the individual characteristics of students. This problem can be solved by using adaptivity by introducing different learning paths.

Theoretical material, as well as material for practical classes and seminars is presented in a different form and volume depending on the learning trajectory.

\section{Training trajectories in the electronic course}

Training using the electronic course can take place along various trajectories. One of the possible options is the introduction of three trajectories: high, medium and low, training on which corresponds to the estimates of "satisfactory", "good" and "excellent."

The distribution along the trajectories is based on the results of the entrance testing. However, in the learning process, students can move along the trajectory of learning, depending on the success of tasks.

Theoretical and practical material on each trajectory is presented in different volumes. After each section on each trajectory of training, it is necessary to pass the final tests. Depending on the test results, several options are possible: - a decrease or increase in the trajectory of training; 
- full-time consultation with the instructor;

Fig. 3 shows the scheme for mastering the discipline

- continuation of the study of the material on this within a single section. trajectory.

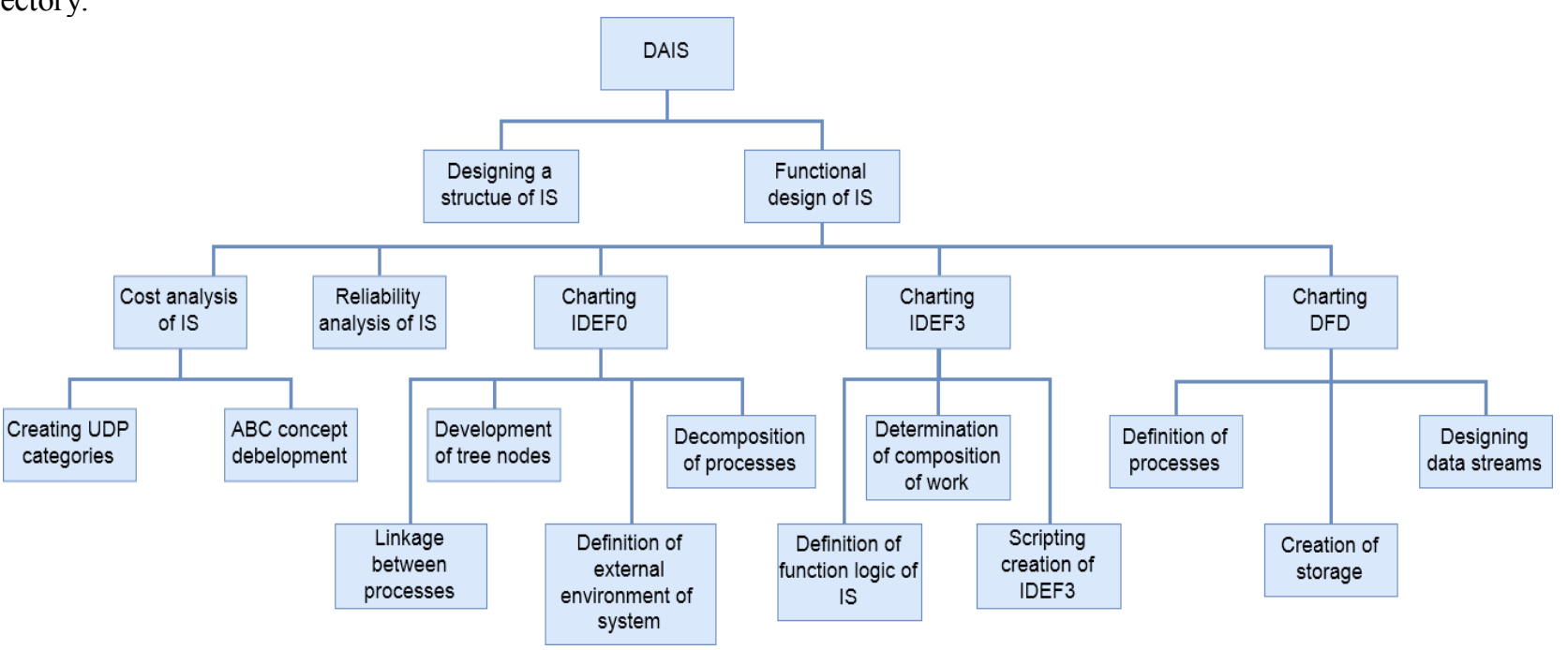

Fig. 2. The adaptive course tree of operations.

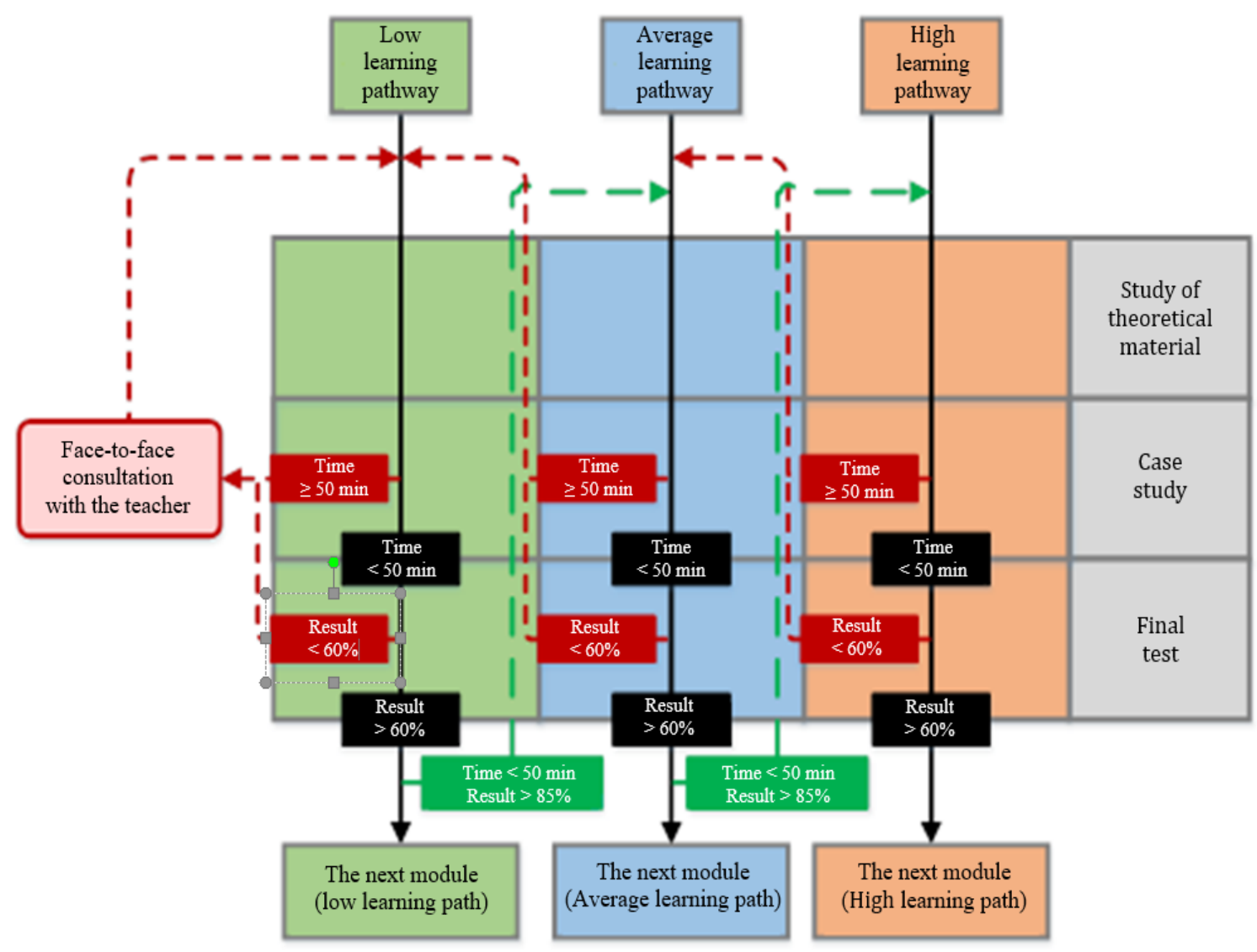

Fig. 3. The scheme for implementing the section in an adaptive electronic course.

After passing the entrance test, if this is the first section, or after passing the test in the previous section, the learner is on one of three trajectories:

- low;

- medium;

- high.
Once again, it should be noted that the theoretical, practical material and the content of the final test on each trajectory are different.

If student has successfully completed more than $85 \%$ of the final test for the material in this section and the test time is less than 50 minutes, then he has the opportunity 
to go to a higher trajectory and, having completed all the required tasks, improve his assessment. However, the student can move to the next section without changing his learning path.

\section{Approbation of adaptive e-learning course}

There was made an analysis of the adaptive e-course effectiveness in teaching students of the Institute of Information and Space Technologies of the Siberian Federal University.

For students of two groups of the third year of "Software Engineering" direction in the adaptive mode, the discipline "Design and architecture of information systems" was conducted.
The number of study groups in which the effectiveness of adaptive courses was conducted was:

- group 1 of the third-year students - 25 people;

- group 2 of the third-year students - 21 people.

The initial distribution along the educational trajectory of the courses was conducted according to the results of the "entrance" testing of knowledge necessary for mastering the corresponding discipline. At the same time, the students had the opportunity of full-time consultation with the teacher for changing the educational trajectory assigned by the system.

The final distribution at the initial stage of training is shown in Fig. 4.

The final grades of students were as follows (Fig. 5).

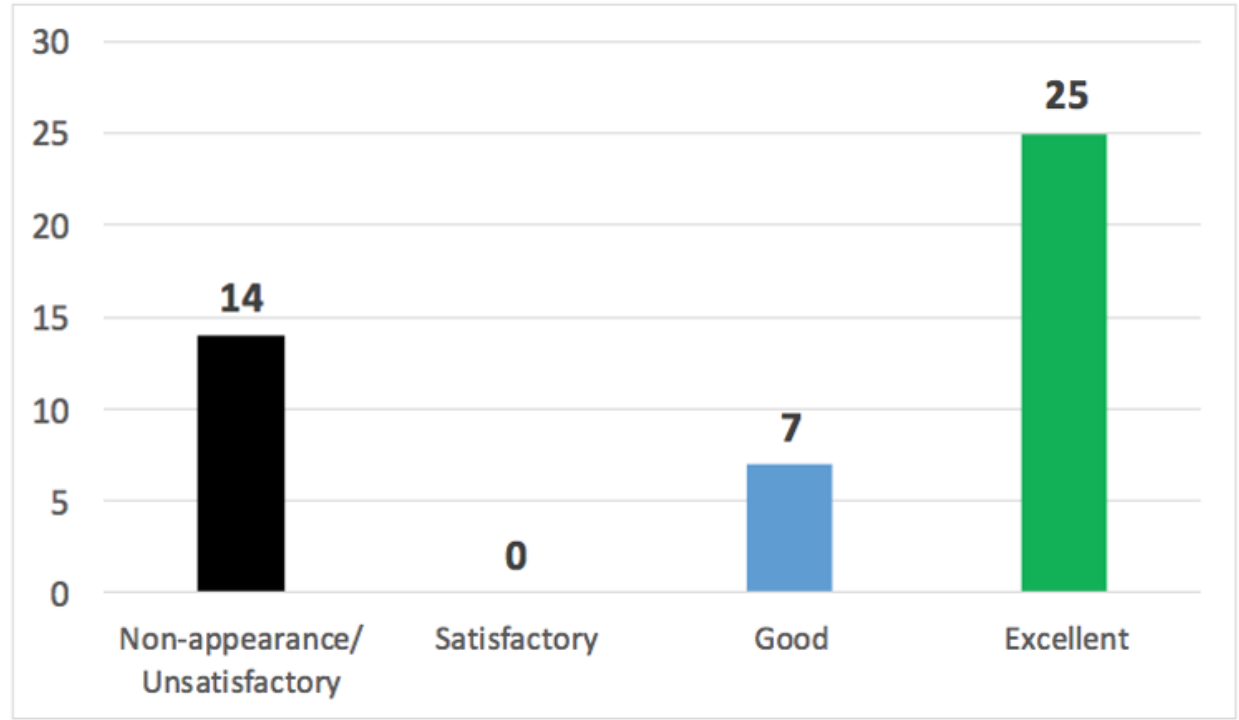

Fig. 4. Distribution of students along the trajectories of the adaptive course at the initial stage of training.

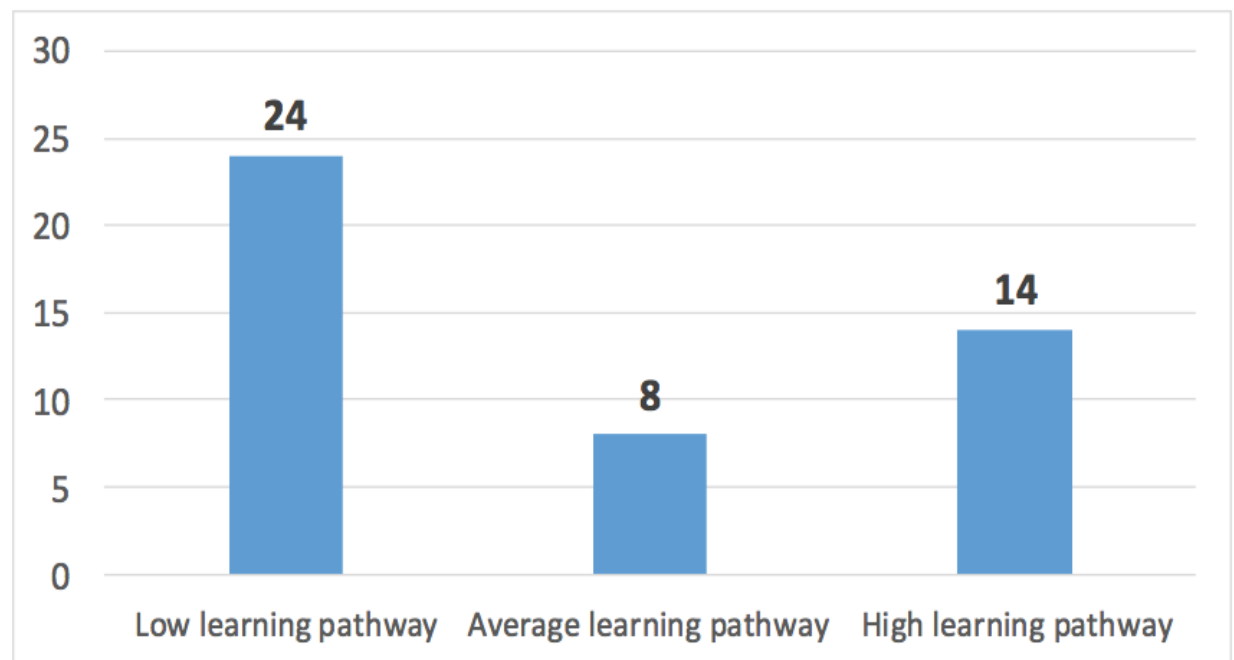

Fig. 5. Final grades of students. 
About half of the students were able to improve their input test results. With the initial distribution of more than $50 \%$ of students to a low trajectory, only $30 \%$ remained at the end of the training. Excellent grades could be obtained by $55 \%$ of students, initially only $30 \%$ of the focus group was distributed on a high trajectory.

\section{Conclusion}

In this article, the development and implementation of an adaptive e-learning course were considered. Adaptivity was realized with the help of training trajectories that correspond to different levels of complexity of the material presented. The distribution along the trajectories is based on the results of the input testing.

In the process of learning, students can navigate the trajectories, making their own schedule of training according to individual preferences and abilities.

\section{References}

1. L.V. Zaytseva, Educational technologies and society 11(1), 135-142 (2008)

2. O. Yu. Zaslavskaya, , O. Ya. Kravets, Bulletin of the Russian University of Peoples' Friendship. Series: Informatization of education 4, 78-92 (2010)

3. A. Solovov Higher education in Russia 11, 301-310 (2006)

4. O. V. Sumsova, Vocational training of students of a technical college in a foreign language: theory and practice, 41-44 (2015)

5. P.V. Sysoev, Language and Culture 4(24), 211-219 (2013)

6. D.A. Sheenok, V.V. Kukartsev, Izvestiya Volgograd State Technical University 14(117), 101-105 (2013)

7. S. He, H. H. Kinshuk, A. Patel, Proceedings of ICALT, 3-7 (2002)

8. O. Antamoshkin, V. Kukarcev, A. Pupkov, R. Tsarev International Multidisciplinary Scientific Conference SGEM 14, 227-232 (2014)

9. W. Hodgins, E. Duval, IEEE 1484.12. 1-2002 (Draft Standard for Learning Object Metadata, 2002)

10. R. C. Clark, R. E. Mayer, E-learning and the science of instruction: Proven guidelines for consumers and designers of multimedia learning. (John Wiley \& Sons, 2016)

11. S. Graf, CELDA 1, 440-443 (2005)

12. A. Paramythis, S. Loidl-Reisinger, Second european conference on e-learning 1, 369-379 (2003)

13. V. Shute, B. Towle, Adaptive e-learning. Educational psychologist 38(2), 105-114 (2003)

14. M. Birjali, A. Beni-Hssane, M. Erritali, Applied Soft Computing Journal 69, 14-32 (2018)

*Corresponding author: vadimond@mail.ru 